\section{Assembling the brain trust: the multidisciplinary imperative in neuro-oncology}

\author{
Ethan B. Ludmir, Anita Mahajan, Verity Ahern, Thankamma Ajithkumar, \\ Claire Alapetite, Valerie Bernier-Chastagner, Ranjit S. Bindra, Andrew J. Bishop, \\ Stephanie Bolle, Paul D. Brown, Christian Carrie, Anthony J. Chalmers (1), \\ Eric L. Chang, Caroline Chung, Karin Dieckmann, Natia Esiashvili, \\ Lorenza Gandola, Amol J. Ghia, Vinai Gondi, David R. Grosshans, \\ Semi B. Harrabi, Gail Horan, Danny J. Indelicato, Rakesh Jalali, \\ Geert O. Janssens, Mechthild Krause, Nadia N. Laack, Normand Laperriere, \\ Anne Laprie, Jing Li, Karen J. Marcus, Susan L. McGovern, Thomas E. Merchant, \\ Kenneth W. Merrell, Laetitia Padovani, Jeannette Parkes, Arnold C. Paulino, \\ Rudolf Schwarz, Helen A. Shih, Luis Souhami, Erik P. Sulman, Roger E. Taylor, \\ Nicola Thorp, Beate Timmermann, Greg Wheeler, Suzanne L. Wolden, \\ Kristina D. Woodhouse, Debra N. Yeboa, Torunn I. Yock, Rolf-Dieter Kortmann \\ and Mary Frances McAleer
}

We read with interest the Position Paper by Aldape and colleagues (Aldape, K. et al. Challenges to curing primary brain tumours. Nat. Rev. Clin. Oncol. https://doi.org/10.1038/ s41571-019-0177-5 (2019) $)^{1}$ on the challenges and future approaches to curing patients with primary brain tumours. We congratulate Cancer Research UK (CRUK) on convening this group of expert clinicians and scientists, and we applaud the authors' elegant synthesis of multiple complex issues. However, we note that among the disciplines represented by the 26 authors of this article, expertise in radiation oncology is conspicuously absent. The authors assert that CRUK "convened an international panel of brain cancer researchers with interests in neurobiology, preclinical tumour modelling, genomics, pharmacology, drug discovery and/or development, neuropathology, neurosurgery, imaging, radiotherapy and medical oncology, with the task of identifying the most important challenges that must be overcome if we are to eventually be in the position to cure all patients with a brain tumour" ${ }^{\prime \prime}$. Aside from radiation oncology, all of the neurooncological subspecialties listed above were represented.

Beyond issues of author representation, radiotherapy is only discussed in the context of efforts to reduce the dose of radiation or to eliminate radiotherapy entirely from the treatment of patients with certain disease characteristics. Even in clinical situations in which attempts to reduce the radiation dose have failed (such as medulloblastoma) ${ }^{2,3}$, the authors contend that this is a function of an historical "inadequate understanding of medulloblastoma biology"1. Without noting the central conclusion of these studies ${ }^{2,3}$ (the importance of adequate irradiation of the neuraxis in patients with medulloblastoma), the authors proceed to discuss why several previous attempts at radiation dose reduction failed and why future efforts will (hypothetically) succeed.

What should already be known to the panel, as well as to members of the broader neuro-oncology community, is that radiotherapy is an integral component of the treatment of brain malignancies. Radiotherapy confers survival advantages to patients with glioblastoma $a^{4,5}$, medulloblastoma ${ }^{2,3}$, germ cell tumours ${ }^{6,7}$, ependymoma ${ }^{8}$ and others ${ }^{9}$. This cost-effective and accessible treatment modality has proven efficacy in the adjuvant setting $^{2-5,8}$, as well as in the definitive setting ${ }^{6,7}$, as a first-line treatment ${ }^{2-8}$ or after prior lines of therapy ${ }^{10}$. Neuro-radiation oncology has witnessed a burgeoning of new techniques, technologies and strategies that will better optimize the therapeutic ratio. For example, stereotactic radiosurgery (SRS) is an increasingly widely used non-operative modality and has provided excellent outcomes with mild toxicities for patients with primary or metastatic intracranial neoplasm $\mathrm{s}^{11}$. Similarly, proton beam therapy (PBT) offers the potential to minimize late-onset toxicities while preserving disease-related outcomes; this promise is increasingly being realized as clinical data on PBT continue to mature ${ }^{12,13}$. Furthermore, multidisciplinary efforts to find synergies between the effects of radiotherapy and novel systemic therapies are ongoing; these collaborative undertakings might translate into more meaningful improvements in survival outcomes ${ }^{14}$. Current and future studies also aim to tailor the delivery of radiotherapy by molecular profile and couple this with high-precision technologies designed to individualize both target volume and dose ${ }^{15}$.

In this sense, the authors of the CRUK position paper $^{1}$ are absolutely correct: the neurooncology community must meet the challenges of treating primary brain tumours with robust research efforts across all boundaries: across disciplines; across geographical borders; across the academia-industry divide; and across the bench-to-bedside spectrum of research. Ensuring that all neuro-oncology disciplines, including radiation oncology, are given a voice as we rise to meet these challenges is imperative to engage in truly collaborative research.

There is a reply to this Correspondence by Aldape, K. et al. Nat. Rev. Clin. Oncol. https:// doi.org/10.1038/s41571-019-0236-y (2019).

Ethan B. Ludmir', Anita Mahajan², Verity Ahern², Thankamma Ajithkumar ${ }^{4}$, Claire Alapetite ${ }^{5}$, Valérie Bernier-Chastagner ${ }^{6}$, Ranjit S. Bindra ${ }^{7}$, Andrew J. Bishop' ${ }^{1}$ Stephanie Bolle ${ }^{8}$, Paul D. Brown', Christian Carrie ${ }^{9}$, Anthony J. Chalmers (iD ${ }^{10}$, Eric L. Chang ${ }^{11}$, Caroline Chung ${ }^{1}$, Karin Dieckmann ${ }^{12}$, Natia Esiashvili' ${ }^{13}$, Lorenza Gandola ${ }^{14}$, Amol J. Ghia', Vinai Gondi ${ }^{15}$, David R. Grosshans' ${ }^{1}$, Semi B. Harrabi ${ }^{16}$,

Gail Horan ${ }^{4}$, Danny J. Indelicato ${ }^{17}$, Rakesh Jalali ${ }^{18}$, Geert O. Janssens ${ }^{19}$, Mechthild Krause ${ }^{20}$, Nadia N. Laack', Normand Laperriere ${ }^{21}$, Anne Laprie ${ }^{22}$, Jing Li', Karen J. Marcus ${ }^{23}$, Susan L. McGovern', Thomas E. Merchant ${ }^{24}$, Kenneth W. Merrell', Laetitia Padovani ${ }^{25}$, Jeannette Parkes ${ }^{26}$,

Arnold C. Paulino ${ }^{1}$, Rudolf Schwarz ${ }^{27}$, Helen A. Shih ${ }^{28}$,

Luis Souhami29, Erik P. Sulman ${ }^{30}$, Roger E. Taylor ${ }^{31}$, Nicola Thorp ${ }^{32}$, Beate Timmermann ${ }^{33}$, Greg Wheeler ${ }^{34}$, Suzanne L. Wolden ${ }^{35}$, Kristina D. Woodhouse', Debra N. Yeboa', Torunn I. Yock ${ }^{28}$, Rolf-

Dieter Kortmann ${ }^{36}$ and Mary Frances McAleer ${ }^{1}$ *

${ }^{\prime}$ Division of Radiation Oncology, The University of Texas MD Anderson Cancer Center, Houston, TX, USA.

${ }^{2}$ Department of Radiation Oncology, Mayo Clinic, Rochester, MN, USA.

${ }^{3}$ Sydney West Radiation Oncology, Crown Princess Mary Cancer Centre, Westmead Hospital, Sydney, New South Wales, Australia.

${ }^{4}$ Department of Oncology, Cambridge University Hospitals, Cambridge, UK.

${ }^{5}$ Department of Radiation Oncology, Institut Curie, Paris and Orsay, France.

${ }^{6}$ Oncology Radiotherapy Institut de Cancérologie de Lorraine, Vandoeuvre les Nancy, France. Department of Therapeutic Radiology, Yale University School of Medicine, New Haven, CT, USA.

${ }^{8}$ Department of Radiation Oncology, Gustave Roussy, Villejuif, France.

${ }^{9}$ Department of Radiation Oncology, Centre Léon Bérard, Lyon, France.

${ }^{10}$ Institute of Cancer Sciences, University of Glasgow, Glasgow, UK.

${ }^{1}$ Department of Radiation Oncology, Keck School of Medicine of USC, Los Angeles, CA, USA. ${ }^{2}$ Department of Radiation Oncology, Universität Klinik für Strahlentherapie und Strahlenbiologie, Vienna, Austria. 
${ }^{13}$ Department of Radiation Oncology, Emory University Winship Cancer Institute, Atlanta, GA, USA ${ }^{14}$ Pediatric Radiotherapy Unit, Fondazione IRCCS Istituto Nazionale dei Tumori, Milan, Italy. ${ }^{15}$ Northwestern University Feinberg School of Medicine, Chicago, IL, USA

${ }^{16}$ Department of Radiation Oncology, University Hospital Heidelberg, Heidelberg, Germany. ${ }^{17}$ Department of Radiation Oncology, University of Florida College of Medicine, Jacksonville, FL, USA

${ }^{8}$ Department of Radiation Oncology, Apollo Proton Cancer Centre, Chennai, Tamil Nadu, India.

${ }^{19}$ Department of Radiation Oncology, University Medical Center Utrecht, Utrecht, Netherlands.

${ }^{20}$ Department of Radiotherapy and Radiation Oncology, Faculty of Medicine and University Hospital Carl Gustav Carus, Technische Universität Dresden Dresden, Germany.

${ }^{21}$ Department of Radiation Oncology, Faculty of Medicine, University of Toronto, Toronto, Ontario, Canada

${ }^{22}$ Department of Radiation Oncology, Institut Claudius Regaud, Institut Universitaire du Cancer de

Toulouse-Oncopole, Toulouse, France.

${ }^{23}$ Department of Radiation Oncology, Dana-Farber Cancer Institute, Boston, MA, USA

${ }^{24}$ Department of Radiation Oncology, St. Jude Children's Research Hospital, Memphis, TN, USA.

${ }^{25}$ Department of Radiation Oncology, Assistance Publique Hôpitaux de Marseille, Marseille, France.

${ }^{26}$ Department of Radiation Oncology, Groote Schuur Hospital and University of Cape Town, Cape Town, South Africa

${ }^{27}$ Department of Radiation Oncology, Medical Center Hamburg-Eppendorf, Hamburg, Germany.

${ }^{28}$ Department of Radiation Oncology, Massachusetts General Hospital, Boston, MA, USA.

${ }^{29}$ Department of Radiation Oncology, McGill University Health Centre, Cedars Cancer Centre, Montréal, Quebec, Canada.

${ }^{30}$ Department of Radiation Oncology, New York University Langone School of Medicine, New York, NY,

${ }^{31}$ College of Medicine, Swansea University, Swansea, UK ${ }^{32}$ Department of Oncology. The Clatterbridge Cancer Centre, Liverpool, UK

${ }^{33}$ Department of Particle Therapy, University Hospital Essen, West German Proton Therapy Centre Essen, West German Cancer Center, German Cancer Consortium, Essen, Germany.

${ }^{34}$ Department of Radiation Oncology and Cancer Imaging, Peter MacCallum Cancer Centre, Melbourne, Victoria, Australia

${ }^{35}$ Department of Radiation Oncology, Memorial Sloan Kettering Cancer Center, New York, NY, USA. ${ }^{36}$ Department of Radiation Oncology, University of Leipzig, Leipzig, Germany.

*e-mail:mfmcalee@mdanderson.org https://doi org/10 1038/s41571-019-0235-z

. Aldape, K. et al. Challenges to curing primary brain tumours. Nat. Rev. Clin. Oncol. https://doi.org/10.1038/ s41571-019-0177-5 (2019).

2. Michalski, J. M. et al. Results of COG ACNS0331: a phase III trial of involved-field radiotherapy (IFRT) and low dose craniospinal irradiation (LD-CSI) with chemotherapy in average-risk medulloblastoma: a report from the Children's Oncology Group. Int. J. Radiat. Oncol. Biol. Phys. 96, 937-938 (2016).

3. Bailey, C. C et al Prospective randomised trial of chemotherapy given before radiotherapy in childhood medulloblastoma. International Society of Paediatric Oncology (SIOP) and the (German) Society of Paediatric Oncology (GPO): SIOP II. Med. Pediatr. Oncol. 25, 166-178 (1995).

4. Walker, M. D. et al. Evaluation of BCNU and/or radiotherapy in the treatment of anaplastic gliomas. A cooperative clinical trial. J. Neurosurg. 49 333-343 (1978).
5. Laperriere, N. et al. Radiotherapy for newly diagnosed malignant glioma in adults: a systematic review. Radiother. Oncol. 64, 259-273 (2002).

6. Balmaceda, C. et al. Chemotherapy without irradiation - a novel approach for newly diagnosed CNS germ cell tumors: results of an international cooperative trial. The first international central nervous system germ cell tumor study. J. Clin. Oncol. 14, 2908-2915 (1996).

7. Rogers, S. J., Mosleh-Shirazi, M. A. \& Saran, F. H. Radiotherapy of localised intracranial germinoma: time to sever historical ties? Lancet Oncol 6, 509-519 (2005).

8. Merchant, T. E. et al. Conformal radiation therapy for pediatric ependymoma, chemotherapy for incompletely resected ependymoma, and observation for completely resected, supratentorial ependymoma. J. Clin. Oncol. 37, 974-983 (2019).

9. Chang, E. L. et al. (eds) Adult CNS Radiation Oncology 1st edn (Springer, 2018)

10. Ludmir, E. B. et al. Increased risk of pseudoprogression among pediatric low-grade glioma patients treated with proton versus photon radiotherapy. Neuro-oncology 21 686-695 (2019)

11. Gilbo, P., Zhang, I. \& Knisely, J. Stereotactic radiosurgery of the brain: a review of common indications. Chin. Clin. Oncol. 6 (Suppl. 2), S14 (2017).

12. Ladra, M. M., MacDonald, S. M. \& Terezakis, S. A. Proton therapy for central nervous system tumors in children. Pediatr. Blood Cancer. 65, e27046 (2018).

13. Indelicato, D. J. et al. Consensus report from the Stockholm Pediatric Proton Therapy Conference. Int. J. Radiat. Oncol. Biol. Phys. 96, 387-392 (2016).

14. Bindra, R. S. et al. GBM radiosensitizers: dead in the water... or just the beginning? J. Neurooncol. 134 , 513-521 (2017).

15. US National Library of Medicine. ClinicalTrials go https://clinicaltrials.gov/ct2/show/record/NCT01878617 (2019).

\section{Reply to 'Assembling the brain trust: the multidisciplinary imperative in neuro-oncology'}

Kenneth Aldape, Kevin M. Brindle, Louis Chesler, Rajesh Chopra, Amar Gajjar,

Mark R. Gilbert, Nicholas Gottardo, David H. Gutmann, Darren Hargrave,

Eric C. Holland, David T. W. Jones, Johanna A. Joyce, Pamela Kearns,

Mark W. Kieran, Ingo K. Mellinghoff, Melinda Merchant, Stefan M. Pfister,

Steven M. Pollard, Vijay Ramaswamy (D), Jeremy N. Rich, Giles W. Robinson (1),

David H. Rowitch, John H. Sampson, Michael D. Taylor, Paul Workman

\section{and Richard J. Gilbertson}

We thank Ludmir and colleagues (Ludmir, E. B. et al. Assembling the brain trust: the multidisciplinary imperative in neuro-oncology. Nat. Rev. Clin. Oncol. https://doi.org/10.1038/ s41571-019-0235-z (2019)) ${ }^{1}$ for their positive comments on our Position Paper (Aldape, K. et al. Challenges to curing primary brain tumours. Nat. Rev. Clin. Oncol. https://doi. org/10.1038/s41571-019-0177-5 (2019)) $)^{2}$, and we agree entirely that radiation oncology has, and will likely continue to have for many years, a critical role in the treatment of patients with primary brain tumours. In particular, we note the helpful references to newer radiotherapy approaches, such as stereotactic radiosurgery and proton beam therapy, that are advancing the treatment of brain tumours. As noted by Ludmir and colleagues $^{1}$, a substantial portion of our Position Paper $^{2}$ is given over to the discussion of radiation oncology.

As stated in our manuscript ${ }^{2}$, this narrative was composed following a series of international, multidisciplinary meetings, hosted by Cancer Research UK, which engaged many individuals involved in brain tumour research and treatment. It is important to note that our manuscript is a Position Paper and not a Meeting Report. Thus, rather than synthesising the entirety of the discussions that took place during these meetings, it reports the opinions of those authors listed on the paper. The discussions held by the committee did indeed engage radiation oncologists as well as individuals in other disciplines; however, other commitments precluded these individuals from participating in many of the meetings and in the writing of our Position Paper $^{2}$. Nonetheless, we wish to assure Ludmir and colleagues ${ }^{1}$ that the entire brain tumour research and treatment community recognizes and values the importance and contribution of radiation oncology to the management of brain tumours. We very much look forward to continuing our interactions with colleagues in this discipline for the good of all patients with these terrible diseases.

Kenneth Aldape ${ }^{1}$, Kevin M. Brindle ${ }^{2}$, Louis Chesler ${ }^{3}$ Rajesh Chopra ${ }^{3}$, Amar Gajjar ${ }^{4}$, Mark R. Gilbert ${ }^{5}$, Nicholas Gottardo ${ }^{6}$, David H. Gutmann Darren Hargrave ${ }^{8}$, Eric C. Holland ${ }^{9}$ David T. W. Jones ${ }^{10}$, Johanna A. Joyce ${ }^{11}$ Pamela Kearns ${ }^{12}$, Mark W. Kieran ${ }^{13}$ Ingo K. Mellinghoff' ${ }^{14}$, Melinda Merchant ${ }^{15}$, Stefan M. Pfister ${ }^{16}$, Steven M. Pollard ${ }^{17}$ Vijay Ramaswamy (DD ${ }^{18}$, Jeremy N. Rich ${ }^{19}$, Giles W. Robinson iD ${ }^{4}$, David H. Rowitch ${ }^{20}$ John H. Sampson ${ }^{21}$, Michael D. Taylor ${ }^{22}$, Paul Workman ${ }^{3}$ and Richard J. Gilbertson iD 2,23* 